\title{
Frecuencia de escoliosis idiopática del adolescente en alumnos de secundaria en seis colegios de Lima Norte
}

Ana Sofía Vázquez-Lazarte ${ }^{1, a}$; Carlos Alberto Berta-Benites 1,a ; Fernando M. Runzer-Colmenares* 1,b

\section{RESUMEN}

Objetivo: Determinar la frecuencia de escoliosis idiopática del adolescente y su asociación con factores como edad y sexo en alumnos de secundaria en seis colegios de Lima Norte.

Materiales y métodos: Se diseñó un estudio observacional, analítico y de corte transversal en seis colegios de la Red Diocesana de Lima Norte. Evaluamos la deformidad de la columna vertebral en alumnos de $1 .^{\circ}$ a $5 .^{\circ}$ año de secundaria de entre 11 a 18 años de edad con la Escala visual de Walter Reed (EVWR). El análisis estadístico se realizó con el software STATA versión 15.0 para Windows, y el análisis bivariado se calculó mediante las técnicas ji cuadrado y t de Student. Un valor de $p \leq 0,05$ fue considerado como significativo.

Resultados: Se incluyeron 191 participantes, 119 varones $(62,30 \%)$ y 72 mujeres $(37,70 \%)$. El promedio de edad fue de 14,46 \pm 1,43 años (rango: 12 - 18 años). Tras la evaluación con la EVWR, 106 participantes (55,50\%) tuvieron un resultado negativo; 33 participantes (17,28 \%), un resultado dudoso, y 52 (27,23\%) tuvieron un resultado positivo. Los hombres representaron el $86,56 \%$ de los resultados positivos y la edad promedio de los participantes fue 14 años.

Conclusiones: Tras la evaluación con la prueba de tamizaje, la frecuencia de escoliosis idiopática del adolescente fue del 27,23 \% (52 participantes). Su asociación con factores como sexo mostró resultados novedosos y la relación con la edad correspondió a lo publicado en estudios previos.

Palabras clave: Escoliosis; Neurocirugía; Pediatría (Fuente: DeCS BIREME).

\section{Frequency of adolescent idiopathic scoliosis among high school students from six Northern Lima schools}

\begin{abstract}
Objective: To determine the frequency of adolescent idiopathic scoliosis and its association with factors such as age and sex among high school students from six Northern Lima schools.

Materials and methods: An observational, analytical and cross-sectional study was conducted in six schools of the Northern Lima Diocesan Network. It assessed the spine deformity among students from the first to fifth years of high school aged between 11 and 18 years old using the Walter Reed Visual Assessment Scale (WRVAS). The statistical analysis was performed using Stata 15 for Windows and the bivariate analysis was conducted using the chi-square test and Student's t-test. A value of $p \leq 0.05$ was considered significant.

Results: A total of 191 participants were included in the study: 119 males $(62.30 \%)$ and 72 females $(37.70 \%)$. The average age was $14.46 \pm 1.43$ years (range: 12 - 18 years). According to the assessment conducted with the WRVAS, 106 participants $(55.50 \%)$ had a negative result, $33(17.28 \%)$ had a dubious result and $52(27.23 \%)$ had a positive result. Males accounted for $86.56 \%$ of the positive results and the average age of the participants was 14 years.

Conclusions: The frequency of adolescent idiopathic scoliosis was $27.23 \%$ ( 52 participants) according to the assessment conducted with the screening test. Its association with factors such as the sex showed novel results, and its relationship with the age matched those published in previous studies.
\end{abstract}

Keywords: Scoliosis; Neurosurgery; Pediatrics (Source: MeSH NLM).

1 Universidad Científica del Sur, Facultad de Ciencias de la Salud, Carrera de Medicina Humana. Lima, Perú.

a Médico, Cirujano General.

b Médico, Epidemiólogo.

* Autor corresponsal. 


\section{INTRODUCCIÓN}

La escoliosis es un defecto tridimensional de la columna vertebral (1-4) que se caracteriza por deformidades en los planos sagital, frontal y transversal ${ }^{(1,2)}$. A su vez, la escoliosis se define como una desviación de la columna en el plano coronal que forma una curvatura mayor que diez grados ${ }^{(2,5,6)}$, que puede diagnosticarse con la medición de las curvas mayores que comprometen la deformidad con el empleo de una radiografía completa de la columna vertebral con el paciente de pie, la cual se realiza con el método de Cobb, lo que da como resultado el ángulo de Cobb ${ }^{(3)}$. Dicha medición determina la gravedad de la escoliosis que puede ser leve $\left(10-20^{\circ}\right)$, moderada $\left(>20-40^{\circ}\right)$ y severa $\left(>40^{\circ}\right)^{(7)}$.

Por otro lado, la escoliosis se puede clasificar en tres tipos: sindrómica, congénita e idiopática (4). La escoliosis congénita se refiere a la deformidad espinal causada por vértebras anormalmente formadas. La escoliosis sindrómica está asociada a trastornos neuromusculares, esqueléticos y enfermedades del tejido conectivo. La escoliosis idiopática es aquella que no tiene una causa identificada y, a su vez, se clasifica según el grupo etario en infantil ( 0 a 3 años), juvenil ( 4 a 10 años) y adolescente (mayores de 10 años) ${ }^{(2,4,5,8)}$, que es la forma más común $(3,8,9)$ y la deformidad espinal más frecuente ${ }^{(2,5)}$. Esta última forma tiene una prevalencia del $1 \%$ al $4 \%$ en adolescentes, y afecta a mujeres jóvenes en la mayoría de los casos ${ }^{(3)}$. En Corea, un estudio prospectivo realizado con un millón de adolescentes, encontró una prevalencia del 3,26\%, la cual era mayor en el sexo femenino, que alcanzó el 4,65\% comparado con $1,97 \%$ en hombres ${ }^{(10)}$.

Diversos estudios nos hablan de factores que influyen en la incidencia de la escoliosis idiopática del adolescente (AIS, por sus siglas en inglés Adolescent Idiopathic Scoliosis). Aseveran que la edad y sexo tienen una influencia positiva sobre esta, y que la AIS es más común en pacientes adolescentes de mayor edad y en mujeres ${ }^{(3,4,8)}$.

Se reconoce una mayor prevalencia de esta enfermedad en el sexo femenino, porque se ha encontrado una relación de AIS con mujeres y hombres que va de 1:1,5 a $3: 1$, que tiene una diferencia mayor conforme aumenta la edad ${ }^{(4,8)}$. Horne et al. reportan que hombres y mujeres tienen el mismo riesgo de desarrollar escoliosis menores de diez grados, pero que las mujeres tienen mayor riesgo de progresión de la deformidad y desarrollo de una enfermedad más severa ${ }^{(8)}$, y se reportan riesgos elevados para curvaturas menores de veinte grados, y mayores aún para casos de curvaturas mayores a cuarenta grados ${ }^{(8)}$.

En relación con la influencia de la edad en la prevalencia de AIS; en Alemania, Konieczny et al. encontraron que la prevalencia ascendía a 11,10 \% en la población entre 14 a
17 años ${ }^{(8)}$. Ya que se han planteado factores relacionados a una mayor prevalencia de AIS, como lo son el sexo y la edad, mediante este estudio buscamos corroborar lo que investigaciones preliminares han demostrado en relación con la frecuencia de escoliosis idiopática en adolescentes y su mayor prevalencia en el sexo femenino y a edades más tardías de la adolescencia.

En el Perú, debido a la falta de estudios estadísticos acerca de esta enfermedad, no es posible conocer la incidencia real de la misma (11), por lo que es necesario extrapolar datos epidemiológicos de otros países. El vació estadístico que se observa y el problema del infradiagnóstico de esta entidad pueden ser completados con el uso de herramientas básicas para el diagnóstico escalonado de la enfermedad. Por ello, se planteó la realización de un estudio observacional y transversal que tuvo como objetivo, encontrar la frecuencia de AIS y los factores asociados a ella, en un sector de la población de Lima, que podría servir como base para futuras investigaciones poblacionales que permitan un mejor conocimiento de la situación epidemiológica de esta enfermedad y difundir las bases para su diagnóstico.

\section{MATERIALES Y MÉTODOS}

\section{Diseño y población de estudio}

Se diseñó un estudio observacional, analítico y de corte transversal que se realizó en colegios de Lima Norte pertenecientes a la Red de Colegios Diocesanos. Los establecimientos fueron el Centro de Educación Primaria (CEP) Diocesano Buen Pastor (localizado en el distrito de Los Olivos y con un nivel socioeconómico A2) ; la Institución Educativa Diocesana Parroquial (IEP) Sagrados Corazones (distrito de Puente Piedra y nivel socioeconómico B); la IEP La Fe de María y la Institución Educativa (IE) Jesús Obrero (localizadas en el distrito de Comas y con niveles socioeconómicos B2 y C1, respectivamente); la IE Nuestra Señora Del Rosario (distrito de Independencia y nivel socioeconómico D); y el IEP Santo Tomás de Valencia (distrito de Santo Rosa de Ancón y nivel socioeconómico D) ${ }^{(12)}$.

El examen fue realizado en ambientes privados proporcionados por cada colegio que participó en el estudio. Las evaluaciones se realizaron en los meses de julio a septiembre del 2018. La muestra se determinó mediante criterios de inclusión y exclusión. Los criterios de inclusión fueron ser alumnos de los colegios seleccionados, cursar entre el $1 .^{\circ}$ al $5 .^{\circ}$ año de secundaria, con una edad entre 11 y 18 años y que aceptararan formar parte del estudio. Los criterios de exclusión fueron no dar la aprobación para ser evaluados, no tener la aprobación de sus padres para participar del estudio y tener antecedes de traumatismo vertebromedular o de cirugía de columna.

El tamaño final de la muestra fue de 191 participantes 
pertenecientes a los seis colegios, tras haber aplicado un muestreo aleatorizado estratificado por cada centro educativo. Sin embargo, la elección de los centros educativos no fue probabilística. La información se registró en una ficha de datos con la Escala visual de Walter Reed (EVWR). Se hizo un cálculo de tamaño muestral considerando el total de alumnos de cada centro educativo. Según el total de alumnos de cada colegio se hizo el cálculo de tamaño muestral estratificando por cada centro educativo y ponderando de la siguiente manera: para el CEP Diocesano El Buen Pastor se consideró un tamaño muestral de 35,00 \% del total; para IE Diocesano Parroquial Sagrados Corazones se calculó 17,00 \%; para la IEP La Fe de María se consideró 8,00\%; para la IE Jesús Obrero fue $24,00 \%$; en la IE Nuestra Señora del Rosario se consideró el $12,00 \%$, y finalmente, para la IEP Santo Tomás de Valencia, se calculó en 4,00\%. Este cálculo se realizó con el programa OpenEpi versión 3.0, de esta manera se incluyó una población total de 277 participantes, y se consideró un probable porcentaje de rechazo estimado del $40,00 \%$. Se invitaron a 388 alumnos a participar en el estudio y se asumió una población infinita y una frecuencia de escoliosis idiopática de $3,26 \%$ con un intervalo de confianza de 99,99\%. El cálculo de tamaño muestral ascendió a 191 participantes ${ }^{(10)}$.

\section{Variables y mediciones}

Presencia de escoliosis idiopática del adolescente: un resultado positivo tras la evaluación con la Escala visual de Walter Reed.

Edad: edad cumplida en años al momento de la evaluación.

Sexo: masculino o femenino.

Nivel socioeconómico: medida que incluye la posición económica y social individual o familiar en relación con otras personas.

Estos niveles están definidos no solo a partir de los ingresos de cada hogar, sino en función de un grupo de variables como nivel educativo alcanzado por el jefe del hogar, sistema de salud al que está afiliado el jefe del hogar, material predominante de la vivienda, conexión del baño, el contar con bienes o servicios como carros particulares o servicio doméstico, el equipamiento del hogar con productos electrónicos y el uso de servicios públicos como cable, internet y teléfono. La información de la categorización socioeconómica fue clasificada en los estratos A, B, C, D, $O E^{(12)}$.

Escala visual de Walter Reed: permite medir la deformidad espinal a partir de siete ítems basados en posturas a examinar ectoscópicamente. Los siete ítems son curvatura corporal, cabeza-pelvis, cabeza-costilla-pelvis, nivel de hombros, rotación escapular, prominencia costal y prominencia del flanco. Estos ítems se encuentran subdivididos en 5 grados que van de menor a mayor intensidad de deformidad (13). Se consideró una evaluación con resultado positivo cuando se presentaba una deformidad compatible con uno o más de los siete ítems contenidos en la EVWR; se consideró un resultado dudoso cuando a la evaluación se le encontraba una alteración discreta no compatible con las alteraciones descritas en la escala; y se consideró negativa cuando no se encontró deformidad alguna.

La recolección de datos fue realizada por los tesistas, alumnos del último año de medicina humana de la Universidad Científica del Sur, y una alumna de la carrera de Tecnología Médica-Terapia Física y Rehabilitación de la Universidad Católica Sedes Sapientiae, quienes previamente fueron capacitados por un neurocirujano especialista en columna.

Luego de obtener la aprobación del Comité de Ética de la Universidad Científica del Sur, se coordinó una reunión con el Obispo de Carabayllo, quien es el promotor de los seis colegios que participaron en el estudio y se autorizó la realización del estudio en los colegios a su cargo. Posteriormente se realizó a una reunión con los docentes de los seis colegios para explicar los fundamentos sobre la escoliosis idiopática del adolescente y la importancia de realizar la investigación, los beneficios y el procedimiento a realizar. Luego se habló con las autoridades de la Universidad Católica Sedes Sapientiae donde se expuso la idea de que los alumnos del último año de la carrera de Tecnología Médica-Terapia Física y Rehabilitación participen en el estudio para ayudar a evaluar a los participantes de los colegios. Se convocó a dos reuniones con los alumnos de dicha Universidad donde fueron capacitados en conocimientos de la enfermedad de estudio y el proceso de la evaluación para la recolección de datos.

Posteriormente, los investigadores se reunieron con los padres de familia de los participantes seleccionados en cada colegio, donde se explicó fundamentos de la enfermedad, la importancia y los beneficios de dicho estudio. En esa misma reunión se entregó el consentimiento informado el cual fue firmado y entregado al equipo de investigadores. Solo fueron reclutados los participantes que firmaron el asentimiento y cuyos padres firmaron el consentimiento informado.

Los datos se recolectaron mediante una ficha creada para llenar la información relevante que incluía la Escala visual de Walter Reed con los siete ítems a evaluar y los cinco grados de cada ítem. Los participantes fueron evaluados con la espalda descubierta (los varones sin polo y las mujeres con una bata que tenía una abertura por detrás). Las participantes fueron evaluadas solo por investigadoras mujeres. Las evaluaciones fueron realizadas por el equipo de investigación en compañía de docentes en un ambiente privado. 
Durante la evaluación, 186 participantes no ingresaron al estudio, pues sus padres no firmaron el consentimiento informado, y 16 participantes no dieron su consentimiento.

\section{Análisis estadístico}

Para el análisis estadístico se utilizó el software STATA versión 15.0 para Windows. Se calcularon frecuencias y porcentajes de las variables categóricas y promedios y desviaciones estándar de las variables numéricas. Luego se realizó un análisis bivariado con la técnica ji cuadrado para analizar variables categóricas y t de Student para análisis de variables categóricas con numéricas. Se consideró un valor de $p \leq 0,05$ como significativos.

Se hizo un análisis bivariado con técnica de ji cuadrado para comparar la proporción de participantes que ingresaron y no ingresaron en el estudio y se halló que no hubo diferencia en sexo ni colegio de origen.

\section{Consideraciones éticas}

El estudio fue autorizado por el Comité de Ética de la Universidad Científica del Sur (Código de registro: 162-2018-PRE15). Se obtuvo la autorización de los participantes y el consentimiento informado de sus padres para formar parte del estudio de manera voluntaria. Los datos recolectados se utilizaron solo para fines de investigación y se manejaron como confidenciales. Los resultados del estudio fueron entregados a los directores de cada colegio. El presente estudio se realizó para el cumplimiento parcial de los requisitos de la obtención del título de Médico Cirujano por parte de los autores Vásquez y Berta.

\section{RESULTADOS}

En el estudio se incluyeron 191 participantes, 119 varones $(62,30 \%)$ y 72 mujeres $(37,70 \%)$, y el promedio de edad fue de 14 años (rango: 12 - 18 años). En la tabla 1 se muestra que la mayoría de los participantes evaluados tuvieron 13 años. La mayor parte de la población fueron varones y cursaban el $2^{\circ}$ grado de secundaria. Respecto a la evaluación con la Escala visual de Walter Reed, 106 participantes $(55,50 \%)$ tuvieron un resultado negativo; 33 participantes $(17,28 \%)$ obtuvieron un resultado dudoso, y $52(27,23 \%)$ tuvieron un resultado positivo para AIS. La mayoría de la población estudiada perteneció al nivel socioeconómico C (Tabla 1).

En relación con el análisis bivariado, el promedio de edad en los participantes que obtuvieron un resultado negativo fue de 14,31 $\pm 1,45$ años; en los que obtuvieron resultado dudoso fue de 14,63 $\pm 1,55$ años, y en los que obtuvieron resultado positivo fue de $14,44 \pm 1,30$ años (Tabla 2 ).

La mayoría de los resultados positivos pertenecen al nivel socioeconómico $\mathrm{C}$, y el nivel socioeconómico que tiene menor número de positivos fue el nivel A (Tabla 3).

La mayoría de resultados positivos fueron varones con un total de 45 participantes (86,58 \%) (Tabla 4).

Tabla 1. Análisis descriptivo de la muestra del estudio

\begin{tabular}{|c|c|c|}
\hline Variables & $\mathrm{n}$ & $\%$ \\
\hline \multicolumn{3}{|l|}{ Edad (años) } \\
\hline 12 & 13 & 6,81 \\
\hline 13 & 52 & 27,23 \\
\hline 14 & 37 & 19,37 \\
\hline 15 & 36 & 18,85 \\
\hline 16 & 41 & 21,47 \\
\hline 17 & 11 & 5,76 \\
\hline 18 & 1 & 0,52 \\
\hline \multicolumn{3}{|l|}{ Sexo } \\
\hline Femenino & 72 & 37,70 \\
\hline Masculino & 119 & 62,30 \\
\hline \multicolumn{3}{|l|}{ Año de estudio } \\
\hline $1 .^{\circ}$ secundaria & 19 & 9,95 \\
\hline $2 .^{\circ}$ secundaria & 54 & 28,27 \\
\hline $3 .^{\circ}$ secundaria & 33 & 17,28 \\
\hline 4. ${ }^{\circ}$ secundaria & 46 & 24,08 \\
\hline $5 .^{\circ}$ secundaria & 39 & 20,42 \\
\hline \multicolumn{3}{|l|}{ Escala } \\
\hline Negativo & 106 & 55,50 \\
\hline Dudoso & 33 & 17,28 \\
\hline Positivo & 52 & 27,23 \\
\hline \multicolumn{3}{|c|}{ Nivel socioeconómico del colegio } \\
\hline A & 37 & 19,37 \\
\hline B & 47 & 24,61 \\
\hline C & 70 & 36,65 \\
\hline D & 37 & 19,37 \\
\hline
\end{tabular}


Tabla 2. Análisis bivariado entre edad y puntaje de escala de Walter Reed

$\begin{array}{lcccc} & & & & \\ \text { Variable } & \text { Negativo Walter Reed } & \text { Pudoso } & \text { Positivo } & \text { Valor de } p \\ \text { Edad } & 14,31 \pm 1,45 & 14,63 \pm 1,55 & 14,44 \pm 1,30 & 0,1\end{array}$

Tabla 3. Análisis bivariado entre nivel socioeconómico y puntaje de escala de Walter Reed

\begin{tabular}{cccc} 
Variable & & Escala Walter Reed & \\
Nivel SE* & Negativo & Dudoso & Positivo \\
\hline A & $24(22,64 \%)$ & $9(27,27 \%)$ & $4(7,69 \%)$ \\
\hline B & $34(32,08 \%)$ & $6(18,18 \%)$ & $7(13,46 \%)$ \\
\hline C & $26(24,53 \%)$ & $13(39,39 \%)$ & $13(59,62 \%)$ \\
D & $22(20,75 \%)$ & $5(15,15 \%)$ & $10(19,23 \%)$ \\
\hline
\end{tabular}

*SE: socioeconómico

Tabla 4. Análisis bivariado entre sexo y puntaje de escala de Walter Reed

\begin{tabular}{cccc} 
Variable & \multicolumn{3}{c}{ Escala Walter Reed } \\
Sexo & Negativo & Dudoso & Positivo \\
\hline Femenino & $51(48,11 \%)$ & $14(42,42 \%)$ & $7(13,46 \%)$ \\
\hline Masculino & $55(51,89 \%)$ & $19(57,58 \%)$ & $45(86,58 \%)$ \\
\hline
\end{tabular}




\section{DISCUSIÓN}

El objetivo del presente estudio fue determinar la frecuencia de escoliosis idiopática del adolescente en una población de participantes de secundaria en seis colegios de Lima Norte. Encontramos que, en la población de 191 participantes, el $27,23 \%$ tuvo un resultado positivo en la prueba de tamizaje de AIS. Esta frecuencia supera a la global, que ha sido descrita en diversos estudios ${ }^{(1-4,8)}$. Este resultado puede ser secundario a que el diagnostico en este estudio se realizó con una prueba de tamizaje, como lo es la Escala visual de Walter Reed. En España, estudios realizados en 2008 y 2014 por Zurita et al. emplearon el test de Adamas como instrumento de medición y diagnóstico de escoliosis, y encontraron prevalencias de $16,00 \%$ y $36,30 \%$, respectivamente. Ambos estudios concluyen que al realizar el diagnóstico mediante pruebas de tamizaje se estima aproximadamente un 40,00 \% de resultados falsos positivos ${ }^{(14,15)}$.

Con relación a la asociación entre la frecuencia de AIS y el sexo, encontramos una mayor frecuencia en el sexo masculino $(86,58 \%)$ con prueba de tamizaje positiva, resultado que difiere a lo que informan los estudios previos ${ }^{(3,4,8)}$.

Aunque la literatura reporta una mayor frecuencia en las mujeres; en Brasil, Souza et al. no encontraron diferencias significativas entre ambos sexos ${ }^{(16)}$. Por otro lado, Zurita et al. encontraron una incidencia de escoliosis del $16,00 \%$ y una afectación mayor en varones que representaba el $57,60 \%$ de la población en un estudio realizado en Granada (España) ${ }^{(14)}$. Si bien este estudio fue realizado en niños de 8 a 12 años, la mayor diferencia de incidencia de acuerdo con el sexo se presenció en el grupo de 12 años ${ }^{(14)}$.

Un factor que pudo influir en que la mayoría de los casos positivos se encuentre en el grupo masculino, fue la mayor cantidad de hombres incluidos en la muestra poblacional. Esta distribución, en la cual encontramos una mayor cantidad de varones en los colegios en comparación con mujeres, coincide con Sánchez Callan en su estudio de escoliosis en una población escolar en un colegio del distrito de La Victoria (Lima) ${ }^{(17)}$. En nuestro estudio, el nivel socioeconómico no mostró una asociación significativa con la escoliosis, este hallazgo se opone a lo que reporta la literatura, según la cual un nivel socioeconómico familiar pobre aparenta estar relacionado a la presencia de la enfermedad ${ }^{(18)}$.

En este estudio se asoció la frecuencia de AIS a factores como género y edad; sin embargo, diferentes estudios demuestran la relación de factores adicionales como edad de inicio de menarquia, índice de masa corporal y genética. Grivas et al. hallaron una relación positiva entre edad tardía de presentación de menarquia y prevalencia de AIS ${ }^{(19)}$ y Pegoraro et al. encontraron una relación positiva entre sobrepeso y obesidad y la escoliosis ${ }^{(20)}$.

Como limitaciones del estudio está el hecho de que se eligieron los colegios por conveniencia, con lo que se tuvo, al inicio, una muestra no probabilística. Sin embargo, se intentó dar validez a la muestra mediante la selección aleatoria de los participantes dentro de la población. Además, la tasa de rechazo fue de 48,60\%, lo que superó la tasa aproximada que se estimó en 40,00\%. Una limitación más de la investigación fue no haber medido el peso y la talla de los participantes, lo que hubiera permitido evaluar el índice de masa corporal como factor de riesgo de escoliosis. Por otro lado, se realizaron charlas a los padres de familia de los participantes, con la finalidad de orientarlos acerca de la enfermedad, invitarlos a formar parte del estudio y solicitar su consentimiento para evaluar a sus hijos menores, obtuvimos una respuesta pobre por parte de estos, ya que solamente el $25 \%$ de los padres asistió a las reuniones.

A pesar de las limitaciones, este sería el primer estudio en el país sobre la frecuencia de escoliosis idiopática del adolescente, y puede servir como punto de partida para trabajos posteriores, en los que pueda efectivizarse la prueba radiográfica para la confirmación del diagnóstico y así hallar una incidencia más objetiva de la enfermedad en nuestro país.

En conclusión, la frecuencia de esta enfermedad en la población estudiada, tras la realización de la evaluación con la prueba de tamizaje, fue cinco veces mayor que los valores encontrados en la revisión bibliográfica, los cuales están en relación con el diagnóstico definitivo de la enfermedad mediante la prueba de patrón de oro. Respecto a la asociación de la escoliosis idiopática del adolescente con el sexo, los resultados fueron novedosos en relación a lo mostrado en la literatura, lo que pudo deberse a la mayor cantidad de hombres evaluados, mientras que la relación con la edad coincidió con estudios previos.

Contribuciones de los autores: Todos los autores contribuyeron en la concepción de la pregunta de investigación, en la redacción, en las conclusiones y en la aprobación de la versión final. Los autores Ana Sofia Vasquez Lazarte y Carlos Berta Benites se encargaron de la recolección de datos y el autor Fernando Runzer Colmenares realizó el análisis de datos estadístico.

Fuentes de Financiamiento: Este artículo ha sido financiado por los autores.

Conflictos de interés: Los autores declaran no tener ningún conflicto de interés. 


\section{REFERENCIAS BIBLIOGRÁFICAS}

1. Kouwenhoven JWM, Castelein RM. The pathogenesis of adolescent idiopathic scoliosis: review of the literature. Spine (Phila Pa 1976). 2008; 33(26): 2898-908.

2. Altaf F, Gibson A, Dannawi Z, Noordeen H. Adolescent idiopathic scoliosis. BMJ. 2013; 346(7906): 1-7

3. Cheng JC, Castelein RM, Chu WC, Danielsson AJ, Dobbs MB, Grivas TB, et al. Adolescent idiopathic scoliosis. Nat Rev Dis Prim. 2015; 1: 15030.

4. Choudhry MN, Ahmad Z, Verma R. Adolescent idiopathic scoliosis. Open Orthop J. 2016; 10(16): 143-54.

5. Hresko MT. Clinical practice. Idiopathic scoliosis in adolescents. $\mathrm{N}$ Engl J Med. 2013; 368(9): 834-41.

6. Jada A, Mackel CE, Hwang SW, Samdani AF, Stephen JH, Bennett JT, et al. Evaluation and management of adolescent idiopathic scoliosis: a review. Neurosurg Focus. 2017; 43(4): E2.

7. Pantoja TS, Chamorro LM. Escoliosis en niños y adolescentes. Rev Méd Clin Las Condes. 2015; 26(1): 99-108.

8. Konieczny MR, Senyurt H, Krauspe R. Epidemiology of adolescent idiopathic scoliosis. J Child Orthop. 2013; 7(1): 3-9.

9. Horne JP, Flannery R, Usman S. Adolescent idiopathic scoliosis: diagnosis and management. Am Fam Physician. 2014; 89(3): 193-8.

10. Suh SW, Modi HN, Yang JH, Hong JY. Idiopathic scoliosis in Korean schoolchildren: a prospective screening study of over 1 million children. Eur Spine J. 2011; 20(7): 1087-94.

11. Nuñez Alvarado LE. Manejo quirúrgico de la escoliosis con instrumentación vertebral posterior en menos de 25 años [Tesis de postgrado]. Lima: Universidad Nacional Mayor de San Marcos. Facultad de Medicina Humana; 2015.

12. APEIM (Asociación Peruana de Empresas de Inteligencia de Mercados). Niveles socioeconómicos [Internet]. 2019. Disponible en: http://apeim.com.pe/2019/08/12/niveles-socio-economicos/

13. Pineda S, Bago J, Gilperez C, Climent JM. Validity of the Walter Reed Visual Assessment Scale to measure subjective perception of spine deformity in patients with idiopathic scoliosis. Scoliosis. 2006; $1: 18$.

14. Zurita Ortega F, Fernández Sánchez M, Fernández García R, Jiménez Schyke CE, Zaleta Morales L. Predictors of scoliosis in school-aged children. Gac Med Mex. 2014; 150(6): 533-9.

15. Zurita Ortega F, Moreno Lorenzo C, Ruiz Rodríguez L, Martínez Martínez A, Zurita Ortega A, Castro Sánchez AM. Cribado de la escoliosis en una población escolar de 8 a 12 años de la provincia de Granada. An Pediatr. 2008; 69(4): 342-50.

16. De Souza Fl, Borges Di Ferreira R, Labres D, Elias R, Miranda de Sousa AP, Pereira RE. Epidemiology of adolescent idiopathic scoliosis in students of the public schools in goiânia-GO. Acta Ortop Bras. 2013; 21(4): 223-5.

17. Sánchez Callán NW. Relación entre escoliosis postural y el índice de masa corporal (IMC) en escolares del nivel primaria de la I. E. $N^{\circ} 1105$ La Sagrada Familia de distrito de La Victoria periodo mayo 2012 - julio 2012. Lima: Universidad Nacional Mayor de San Marcos. Facultad de Medicina Humana; 2012.

18. Rocha JCT, Tatmatsu DIB, Vilela DA. Associação entre uso de mochilas escolares e escoliose em adolescentes de escolas públicas e privadas. Motricidade. 2012; 8(Suppl. 2): 803-9.

19. Grivas TB, Vasiliadis E, Mouzakis V, Mihas C, Koufopoulos G. Association between adolescent idiopathic scoliosis prevalence and age at menarche in different geographic latitudes. Scoliosis. 2006; 1: 9.

20. Pegoraro Baroni M, Bouzas Sanchis GJ, Costa de Assis SJ, Gomes dos Santos R, Alves Pereira S, Galante Sousa K, et al. Factors associated with scoliosis in schoolchildren: A cross sectional population-based study. J Epidemiol. 2015; 25(3): 212-20.
Correspondencia:

Carlos Alberto Berta Benites

Dirección: Jr. Gnral. Canterac 152 dpto 202. Jesús María, Perú.

Teléfono: 999222458

Correo electrónico: Hcarlosbh@gmail.com

Recibido: 13 de febrero de 2020

Evaluado: 26 de agosto de 2020

Aprobado: 05 de setiembre de 2020

(c) La revista. Publicado por Universidad de San Martín de Porres, Perú. (c) bajo términos de Licencia Creative Commons Atribución 4.0 Internacional. (http://creativecommons.org/licenses/by/4.0/)

\section{ORCID iDs}

Ana Sofía Vázquez-Lazarte Cichttps://orcid.org/0000-0002-9243-1216

Carlos Alberto Berta-Benites ㄱ https://orcid.org/0000-0002-7243-5041

Fernando M. Runzer-Colmenares https: / / orcid.org/0000-0002-7717-8996 